\title{
Double-wavelet approach to study frequency and amplitude modulation in renal autoregulation
}

Sosnovtseva, Olga; Pavlov, A.N.; Mosekilde, Erik; Holstein-Rathlou, N.-H.; Marsh, D.J.

Published in:

Physical Review E

Link to article, DOI:

10.1103/PhysRevE.70.031915

Publication date:

2004

Document Version

Publisher's PDF, also known as Version of record

Link back to DTU Orbit

Citation (APA):

Sosnovtseva, O., Pavlov, A. N., Mosekilde, E., Holstein-Rathlou, N-H., \& Marsh, D. J. (2004). Double-wavelet approach to study frequency and amplitude modulation in renal autoregulation. Physical Review E, 70(3), 031915. https://doi.org/10.1103/PhysRevE.70.031915

\section{General rights}

Copyright and moral rights for the publications made accessible in the public portal are retained by the authors and/or other copyright owners and it is a condition of accessing publications that users recognise and abide by the legal requirements associated with these rights.

- Users may download and print one copy of any publication from the public portal for the purpose of private study or research.

- You may not further distribute the material or use it for any profit-making activity or commercial gain

- You may freely distribute the URL identifying the publication in the public portal 


\title{
Double-wavelet approach to study frequency and amplitude modulation in renal autoregulation
}

\author{
O. V. Sosnovtseva, ${ }^{1}$ A. N. Pavlov ${ }^{2}$ E. Mosekilde, ${ }^{1}$ N.-H. Holstein-Rathlou, ${ }^{3}$ and D. J. Marsh ${ }^{4}$ \\ ${ }^{1}$ Department of Physics, The Technical University of Denmark, 2800 Kongens Lyngby, Denmark \\ ${ }^{2}$ Department of Physics, Saratov State University, Astrakhanskaya Str. 83, Saratov, 410026, Russia \\ ${ }^{3}$ Department of Medical Physiology, University of Copenhagen, 2200 Copenhagen N, Denmark \\ ${ }^{4}$ Department of Molecular Pharmacology, Physiology, and Biotechnology, Brown University, Providence, Rhode Island 02912, USA
}

(Received 10 March 2004; published 30 September 2004)

\begin{abstract}
Biological time series often display complex oscillations with several interacting rhythmic components. Renal autoregulation, for instance, involves at least two separate mechanisms both of which can produce oscillatory variations in the pressures and flows of the individual nephrons. Using double-wavelet analysis we propose a method to examine how the instantaneous frequency and amplitude of a fast mode is modulated by the presence of a slower mode. Our method is applied both to experimental data from normotensive and hypertensive rats showing different oscillatory patterns and to simulation results obtained from a physiologically based model of the nephron pressure and flow control. We reveal a nonlinear interaction between the two mechanisms that regulate the renal blood flow in the form of frequency and amplitude modulation of the myogenic oscillations.
\end{abstract}

DOI: 10.1103/PhysRevE.70.031915

PACS number(s): 87.19. - j, 87.90.+y, 05.45.Tp

\section{INTRODUCTION}

Living systems often generate signals that reflect the coexistence of rhythmic components originating in different mechanisms. Besides the heart beat, analyses of blood pressure data, for instance, are likely to reveal components produced by both the respiratory cycle and the vasomotoric activity of small arteries and arterioles [1,2]. The presence of such multimode dynamics is well-documented for physiological control systems at the cellular or subcellular level (e.g., the bursting activity of pancreatic $\beta$ cells [3] and of certain nerve cells $[4,5]$ ) and the systemic level (e.g., the coexistence of a $24 \mathrm{~h}$ circadian rhythm and a 2-4 h ultradian rhythm in insulin secretion [6]).

Interaction between coexisting modes can lead to a variety of interesting nonlinear dynamic phenomena, with entrainment or synchronization representing the most prominent example. Experimental and/or numerical investigations of synchronization have been performed for many different physiological systems, including, for instance, the cardiorespiratory system [7] and small ensembles of nerve cells [8]. However, even when synchronization does not occur, the mode-to-mode interaction may reveal itself in the form of modulation. The instantaneous amplitude or frequency of one mode may be modulated by the presence of the other [9].

Experimentally it may be possible to record only a single variable (such as the blood pressure or the concentration of a certain hormone), and the question here arises: to what extent will it be possible to demonstrate and characterize the modulation of a fast mode from a time series that is dominated by a slower mode? It is the purpose of the present paper to illustrate how one can address this question by means of double-wavelet analysis. The method will be applied to analyze tubular pressure and blood flow data from rat nephrons in order to understand the interaction between the slower tubuloglomerular feedback and the faster myogenic mechanism in renal autoregulation.

At the level of the individual functional unit (the nephron), renal pressure and flow control involves two different mechanisms: the tubuloglomerular feedback (TGF), which regulates the incoming blood flow in response to variations of the $\mathrm{NaCl}$ concentration of the tubular fluid near the terminal point of the loop of Henle, and a myogenic mechanism by which the afferent arteriole regulates its diameter in response to variations in its transmural pressure. Both of these mechanisms can produce rhythmic variations, and interaction between the two signals can lead to various modes of intranephron synchronization [10-12].

Investigation of nephron dynamics is most readily performed by measuring the hydrostatic pressure in proximal tubules of superficial nephrons (i.e., nephrons close to the surface of the kidney). This signal is dominated by the relatively slow TGF oscillations. We shall show, however, how tubular pressure variations can be used to obtain information about the modulation of the frequency and amplitude of the faster myogenic oscillations and, hence, about how the slower mode influences the faster one.

Physiological processes are often highly nonstationary. Part of this nonstationarity may be ascribed to changing environmental conditions or to interactions with other regulatory processes. It is known, however, that the degree of nonstationarity can differ between states of health and disease [13]. Because of their nonstationarity, analyses of physiological time series often apply the concept of slowly varying parameters; it is assumed that the statistical characteristics of the data remain practically constant during certain time intervals, and the analysis is performed by using a sliding window. This approach is useful if the nonstationarity is associated with low-frequency spectral components relative to the physiological rhythms of interest. If the properties of the experimental data display essential variations over short time intervals, one has to apply specialized tools such as wavelet analysis $[14,15]$, detrended fluctuation analysis [16], or other techniques [17].

A first step in our analysis is to separate the slow and the fast oscillatory modes. A way to approach this is to apply band pass filtering, although in nonlinear systems this proce- 
dure may fail to correctly separate the different modes in the presence of harmonics and interaction frequencies in the spectrum. Nonstationarity can also create problems in a numerical study of modulation because the modulation properties can change in response to changes of the system parameters. In particular, if the instantaneous frequency of a mode changes in time, the choice of filter parameters becomes a matter of serious concern. On the one hand, one cannot apply a narrow frequency range for filtering because the rhythms float. On the other hand, if the two modes are close enough in the frequency domain, one cannot use a broad frequency range in the band pass filter since in that case the rhythms may not be separated. Such problems can sometimes be solved by using a sliding-window analysis [18] or, as we shall demonstrate later, different aspects of multimode dynamics can be studied with wavelets.

\section{DOUBLE-WAVELET TECHNIQUE}

Spectral analyses of biological time series are often based on the application of a wavelet transformation [14,15]. The advantages of this approach in comparison with the classical Fourier transform have been widely discussed. The wavelet transform of a signal $x(t)$ is obtained as follows:

$$
T_{x}(a, t)=\frac{1}{\sqrt{a}} \int_{-\infty}^{\infty} x(u) \psi^{*}\left(\frac{u-t}{a}\right) d u .
$$

Here $\psi$ is a "mother" function that should be soliton-like with zero average. $T_{x}(a, t)$ are the wavelet coefficients and $a$ is a time scale parameter. The details of this transform (e.g., the choice of $\psi$ ) depend on the problem to be solved. In the analysis of rhythmic components, the Morlet function is typically considered. Instead of the Morlet wavelet, some authors [19] prefer to use other complex wavelet functions because of possible spurious effects, especially for time series with nonzero mean. To avoid such effects we have transformed all analyzed time series to zero mean value before applying the wavelet technique. By performing an analysis of nephron dynamics, we have checked and found that there is practically no difference between the results obtained with the Morlet wavelet and with the function used in [19]. A simplified expression of the Morlet function has the form

$$
\psi(\tau)=\pi^{-1 / 4} \exp \left(j 2 \pi f_{0} \tau\right) \exp \left[-\frac{\tau^{2}}{2}\right] .
$$

The value $f_{0}$ allows us to search for a compromise between the localizations of the wavelet in the time and frequency domains. In our work $f_{0}=1$. The relation between the scale $a$ and the central frequency for the mother function $f$ in this situation is $f=1 / a$.

Besides the coefficients $T_{x}(a, t)$, the energy density of the signal $x(t)$ in the time scale plane can be estimated: $E_{x}(a, t)$ $\sim\left|T_{x}(a, t)\right|^{2}$. Following the definition used in Ref. [15], the coefficient of proportionality between $E_{x}(a, t)$ and $\left|T_{x}(a, t)\right|^{2}$ depends on both the scale and the shape of the mother wavelet although in some works the simpler expression $\left[E_{x}(a, t)\right.$ $\left.=\left|T_{x}(a, t)\right|^{2}\right]$ is considered. Note that the modulus of the original wavelet coefficients $T_{x}(a, t)$ estimated from Eq. (1) do not correspond to actual amplitudes of rhythmic components. To study amplitude variations, it is possible to slightly change the definition of the wavelet transform [17] or to make corrections for the energy density $E_{x}(a, t)$. In our work we consider $E_{x}(a, t)=C a^{-1}\left|T_{x}(a, t)\right|^{2}$, where $C$ is a parameter that depends on the wavelet mother function.

$E_{x}(a, t)$ represents a surface in three-dimensional space whose sections at fixed time moments correspond to the local energy spectrum. To simplify the visualization of this surface, the dynamics of only the local maxima of $E_{x}(a, t)$ or $E_{x}(f, t)$, i.e., the time evolution of the spectral peaks will be considered. Figure 1(b) demonstrates all maxima of $E_{x}(f, t)$ detected in the original signal [Fig. 1(a)] at each time moment $t$ (i.e., we consider different components irrespective of their contributions). However, this type of figure does not give information about the dominant spectral components. Such information can be obtained, for example, from a scalogram, i.e., a time averaged power spectrum, which is an analogue to the Fourier power spectrum. Such a scalogram is illustrated in Fig. 1(c) where a well-pronounced peak around $0.033 \mathrm{~Hz}$, corresponding to the slow mode, is distinguishable. The other peak at $0.15-0.17 \mathrm{~Hz}$ derives from the fast myogenic dynamics. Since both frequency components are of physiological interest we extract them from the original wavelet transformation for further analysis of their properties. Figure 1(d) displays the temporal behavior of the local spectral peaks associated with the slow $\left(f_{\text {slow }}\right)$ and the fast $\left(f_{\text {fast }}\right)$ oscillatory modes in the dynamics of the nephron [Fig. 1(a)]. For the considered example, both modes are wellexpressed in the two-dimensional representation of $E_{x}(f, t)$. In many cases, as discussed in Sec. III, the fast mode displays strong nonstationarity.

It can be seen that $f_{\text {slow }}$ remains practically constant while $f_{\text {fast }}$ changes in time. Moreover, since Fig. 1(d) only represents the visualization of the surface $E_{x}(f, t)$, a value of the energy density is associated with each point shown in this figure. This means that we can extract the time dependences of the instantaneous frequencies $f_{\text {slow }}(t)$ and $f_{\text {fast }}(t)$ from $E_{x}(f, t)$ as well as the dependences of the instantaneous values of the energy densities $e_{\text {slow }}(t)$ and $e_{\text {fast }}(t)$. Or, considering the energy as a quantity that varies proportional to the square of the amplitude, it is possible to characterize the dynamics in terms of the instantaneous amplitudes.

Aiming to study modulation properties of the fast mode, we propose to use the following approach. The time dependence of the instantaneous frequency $f_{\text {fast }}(t)$ is considered as input signal for the next wavelet transform (1). Again, the wavelet coefficients and the energy density are estimated and the simplified visualization of the energy density is considered. The latter will contain information about all modes involved in the modulation process. In the case of nonstationary dynamics we can examine how the features (characteristics) of the frequency modulation are changed in time. By analogy, instead of the instantaneous frequency of the fast dynamics we can take the instantaneous amplitude of this oscillatory mode and, therefore, it is possible to study the properties of amplitude modulation of the fast rhythm as well. 

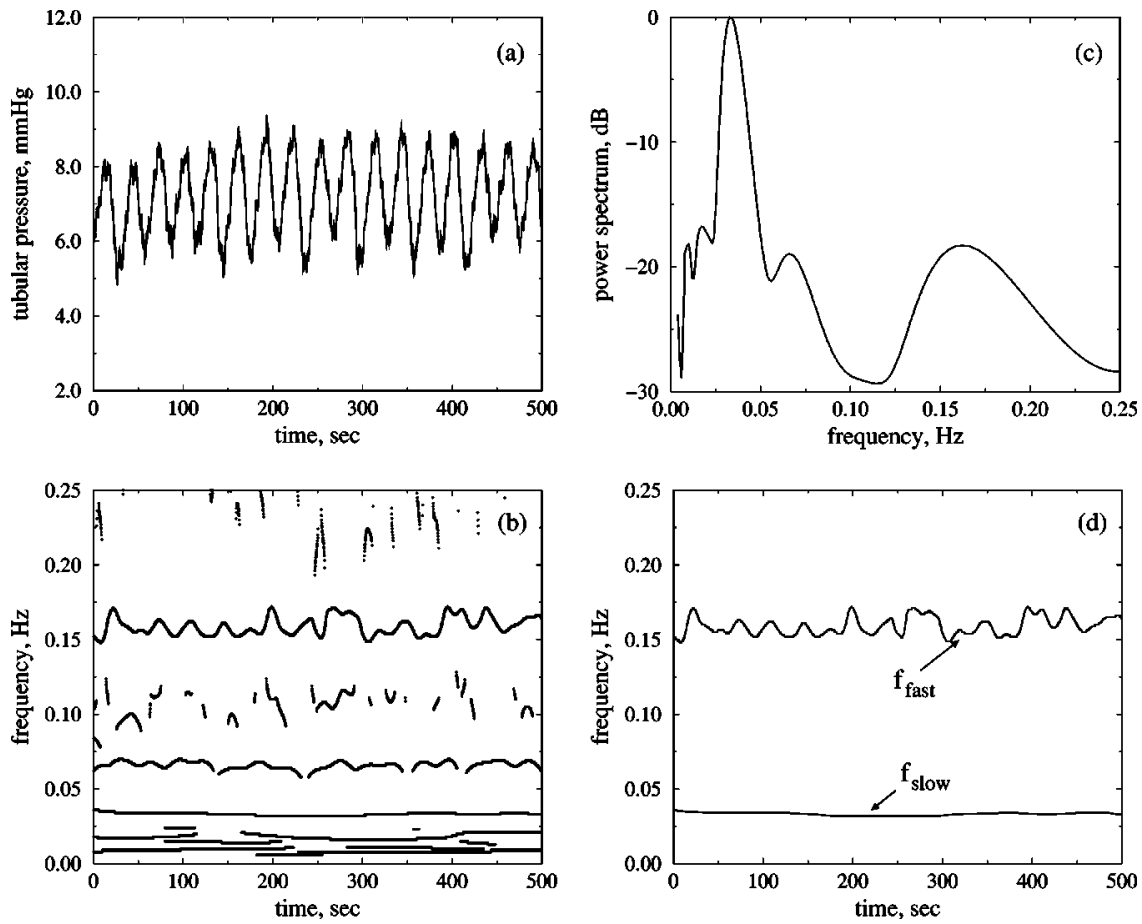

FIG. 1. Experimental recording of the proximal tubular pressure in a single nephron of a rat kidney (a) and results of its wavelet analysis: the dynamics of all local maxima of the energy density (b), the scalogram (c) and the extracted frequencies of rhythmic components (d). The search for $f_{\text {slow }}$ and $f_{\text {fast }}$ was performed in the ranges $0.02-0.07$ and $0.1-0.25 \mathrm{~Hz}$, respectively. The frequency step in the wavelet transform was chosen to be $0.001 \mathrm{~Hz}$.
This approach, which we shall refer to as a doublewavelet analysis, allows us to characterize the nonstationary temporal dynamics of a modulated signal, i.e., to detect all components that are involved in the modulation, estimate their contributions, and analyze whether the modulation properties change during the observation time. Figure 2 demonstrates the results of such an analysis for the time series shown in Fig. 1(a). We can see that the modulation frequency (white circles) is close to the slow rhythm (black circles) for both the frequency and amplitude modulations [Figs. 2(a) and 2(b), respectively]. The slow mode may not be the only component detected in the modulation process. In particular, the spectrum of modulation can contain harmonics of $f_{\text {slow }}$ and/or a low-dimensional structure similar to $1 / f$ dynamics.

\section{INTERACTING FAST AND SLOW MODES}

\section{A. Mathematical model of a single nephron}

As mentioned in the introduction, the individual functional unit of the kidney (the nephron) displays bimodal oscillations in its regulatory function. The nephron may be considered as a filtration device with an internal feedback (the so-called tubuloglomerular feedback) that regulates the incoming blood flow depending on the $\mathrm{NaCl}$ concentration of the fluid that leaves the loop of Henle [20-22]. Experiments by Leyssac and Holstein-Rathlou [20,21] have demonstrated that this feedback regulation can become unstable and generate self-sustained oscillations in the proximal intratubular pressure with a typical period of $30-40 \mathrm{~s}$ (slow mode). These oscillations are typically regular for normal rats and irregular for rats with a genetic form of high blood pressure, so-called spontaneously hypertensive rats [20]. The second (fast) mode is connected with a myogenic mechanism associated with an intrinsic response of the smooth muscle cells
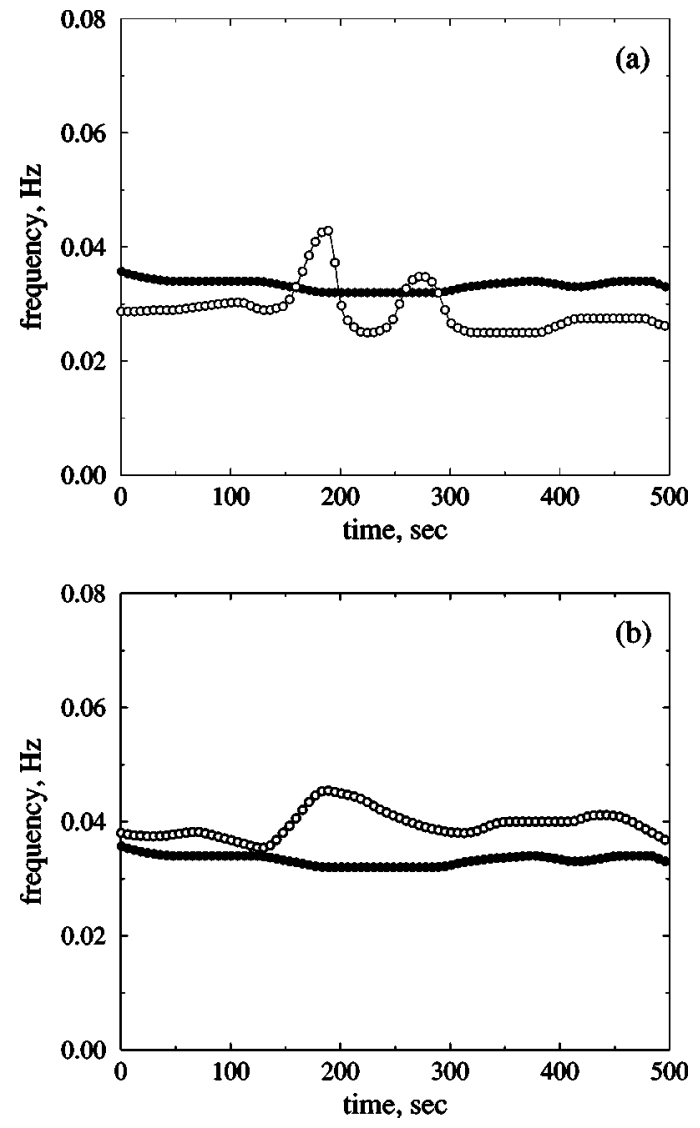

FIG. 2. Results of the double-wavelet analysis of the time series shown in Fig. 1(a). (a) and (b) correspond to the cases of frequency and amplitude modulation, respectively. Black circles mark $f_{\text {slow }}(t)$ and white circles indicate the instantaneous modulation frequency. 

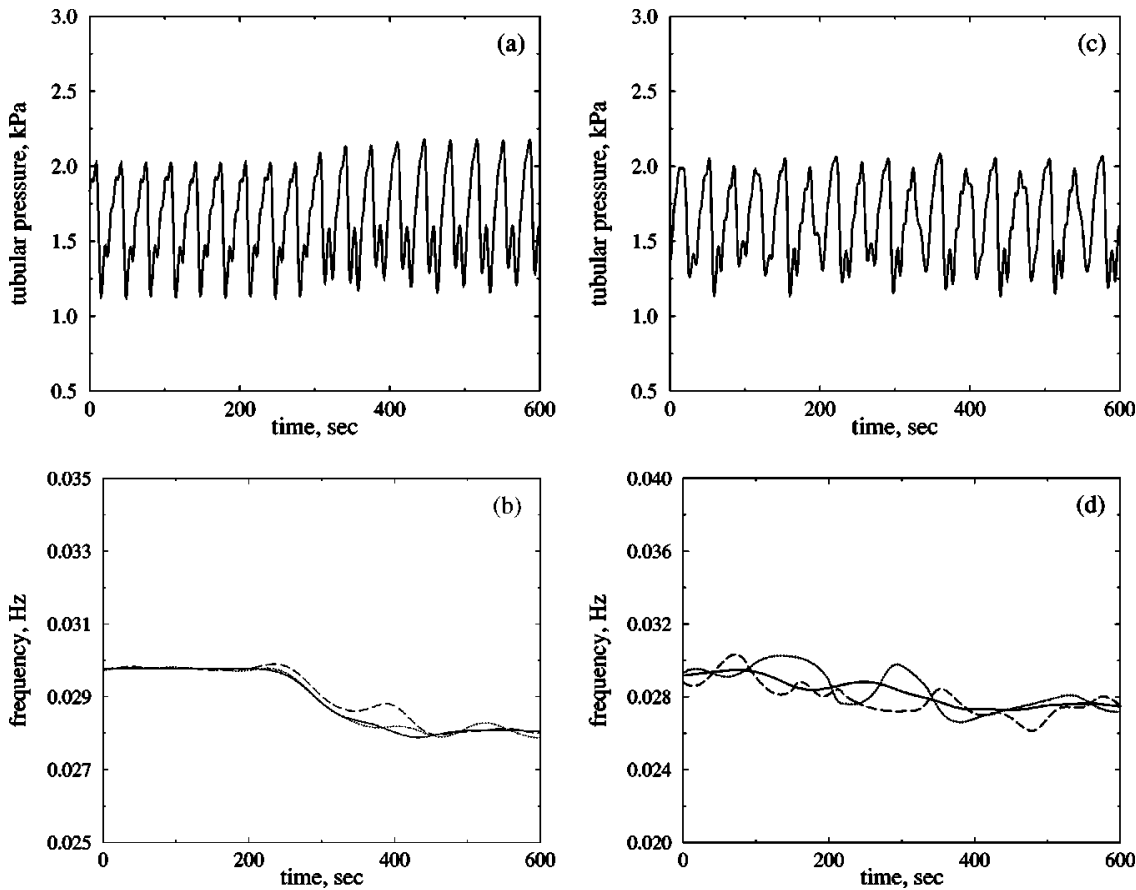

FIG. 3. Numerical analysis of the mathematical model (3). Responses to a parameter change of the tubular pressure and modulation properties in the periodic $(a, b)$ and chaotic $(c, d)$ regimes, respectively. Solid curves in $(b, d)$ correspond to the instantaneous frequency of the slow mode. The instantaneous modulation frequencies are presented by dashed curves (the case of amplitude modulation) and by dotted curves (the case of frequency modulation). The parameters for the wavelet analysis are the same as in Fig. 1. in the vascular wall of the afferent arteriole. When excited by the slow oscillations, this mechanism produces oscillations with a period of about 5-10 s.

The main structure of the nephron model is discussed, for instance, in Refs. [9,23,24]. The autoregulation of the blood flow to the individual nephron may be described by the six ordinary differential equations

$$
\begin{gathered}
\dot{P}_{t}=\frac{1}{C_{\text {tub }}}\left[F_{f}\left(P_{t}, P_{a}, r\right)-F_{\text {reab }}-\left(P_{t}-P_{d}\right) / R_{H}\right], \\
\dot{r}=v_{r}, \\
\dot{v}_{r}=\frac{1}{\omega}\left[P_{\mathrm{av}}\left(P_{t}, P_{a}, r\right)-P_{\mathrm{eq}}\left(r, \Psi\left(X_{3}, \alpha\right), T\right)-\omega d v_{r}\right], \\
\dot{X}_{1}=\frac{1}{R_{H}}\left(P_{t}-P_{d}\right)-\frac{3}{T} X_{1}, \\
\dot{X}_{2}=\frac{3}{T}\left(X_{1}-X_{2}\right), \\
\dot{X}_{3}=\frac{3}{T}\left(X_{2}-X_{3}\right) .
\end{gathered}
$$

The first equation determines the pressure variations in the proximal tubule in terms of the in- and outgoing fluid flows. Here, $F_{f}$ is the single-nephron glomerular filtration rate and $C_{\text {tub }}$ is the elastic compliance of the tubule. The flow into the loop of Henle is given by the difference between the proximal and the distal tubular pressures and by the flow resistance $R_{H}$. The reabsorption in the proximal tubule $F_{\text {reab }}$ is assumed to be constant. The arterial blood pressure $P_{a}$ is used as a control parameter in our simulations.
The next two equations describe the dynamics associated with the flow control in the afferent arteriole. Here, $r$ represents the radius of the active part of the vessel and $v_{r}$ is its rate of increase. $d$ is a characteristic time constant describing the damping of the oscillations, $\omega$ is a measure of the mass density of the arteriolar wall, and $P_{\text {av }}$ denotes the average pressure in the active part of the arteriole. $P_{\mathrm{eq}}$ is the value of this pressure for which the arteriole is in equilibrium with its present radius and muscular activation $\Psi$. The expressions for $F_{f}, P_{\mathrm{av}}$, and $P_{\mathrm{eq}}$ involve a number of algebraic equations that must be solved along with the integration of Eqs. (3).

The remaining equations in the single-nephron model represent the delay $T$ in the TGF regulation. This delay arises both from the transit time through the loop of Henle and from the cascaded enzymatic processes between the macula densa cells and the smooth muscle cells that control the contractions of the afferent arteriole. For a more detailed explanation of the model and its parameters, see, e.g., Ref. [23].

The model (3) represents a relatively accurate account of the basic physiological mechanisms responsible for the regular oscillations in normotensive rats as well as for the chaotic dynamics observed in spontaneously hypertensive rats. Over the years it has been tested and examined in many different ways.

With this model we can demonstrate the possibility of extracting information about the modulation properties of the fast oscillatory mode from the slow phase variable $P_{t}$. For this purpose we consider two different dynamical regimes, namely periodic oscillations for $\alpha=26$ and chaotic oscillations for $\alpha=28$ [Figs. 3(a) and 3(c)]. To show the efficiency of the proposed approach in the case of nonstationary dynamics we have performed a stepwise change of a selected control parameter: The parameter $P_{a}$ characterizing the arterial pressure is changed from $P_{a}=13 \mathrm{kPa}$ to $P_{a}=13.3 \mathrm{kPa}$ at $t=300 \mathrm{~s}$ for the signal shown in Fig. 3(a), and the parameter $\alpha$ is changed from $\alpha=28$ to $\alpha=31$ at the same time moment for the process considered in Fig. 3(c). 

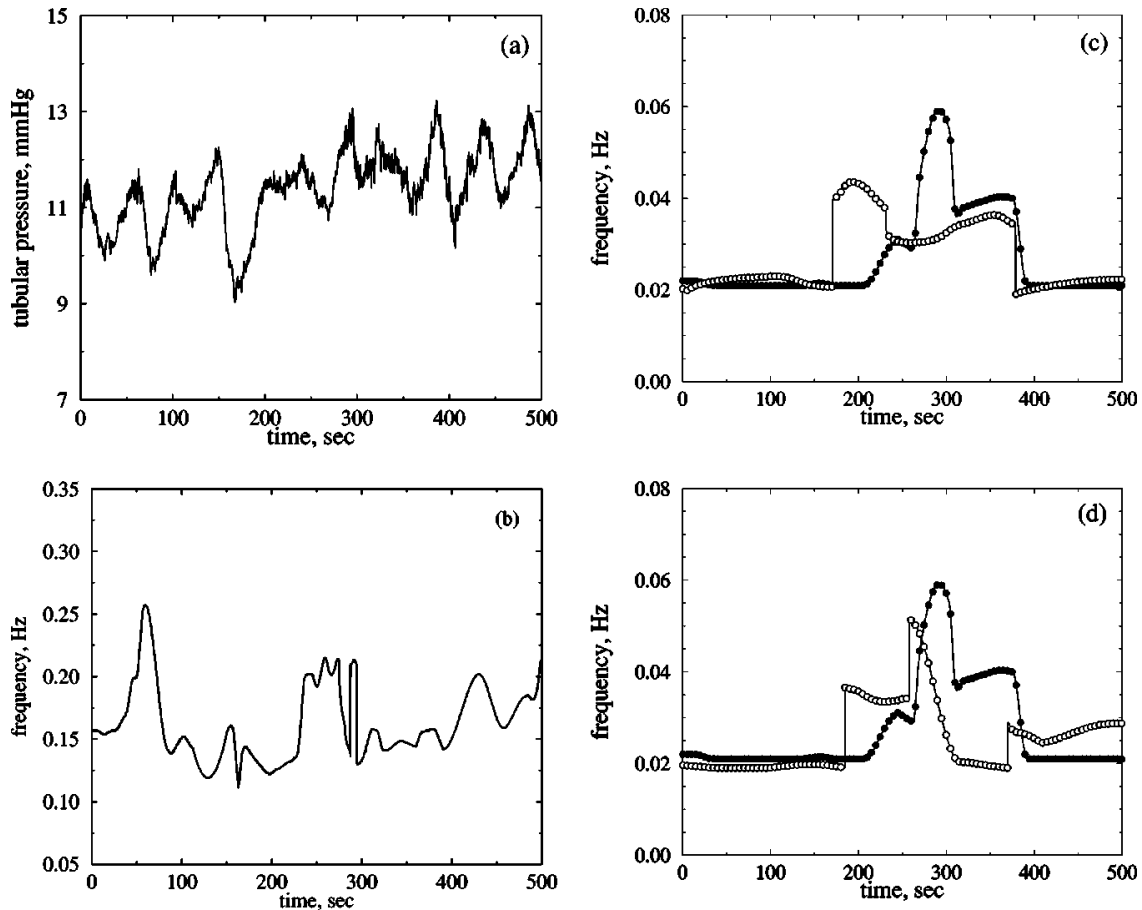

FIG. 4. Analysis of the tubular pressure data for a hypertensive rat. (a) is the original time series. (b) demonstrates the instantaneous frequency of the fast mode $f_{\text {fast }}(t)$. (c) and (d) show the instantaneous frequency of the slow mode (black circles) and the modulation frequency (white circles) for the frequency and amplitude modulation of the fast mode, respectively.
Various tests performed with the nephron model and with different simpler examples of modulation (such as a multiplicative interaction modeled by two harmonic functions) have shown that the presence of a slow nonstationarity, such as a variation of the arterial blood pressure with its $1 / f$-spectral properties, does not have an essential influence on our ability to estimate the modulation frequency.

The results of our numerical investigations are presented in Figs. 3(b) and 3(d). Here, the solid curves correspond to the instantaneous frequencies of the slow oscillatory mode (detected by analogy with Fig. 1). The instantaneous frequencies of the amplitude modulation (dashed) and of the frequency modulation (dotted) are obtained with the doublewavelet analysis introduced in the previous section. Note, that the modulation frequencies demonstrate a stronger floating around the slow rhythm in the chaotic regime. These fluctuations can probably be reduced by taking longer data with a better resolution. We decided, however, to restrict our findings to time series of the same length as available in our typical experimental recordings (4096 points).

\section{B. Experimental results}

Despite the emergence of a consensus on the combined participation of both the myogenic and the TGF mechanisms in autoregulation, there are only a few attempts to examine the interplay between them.

Let us consider recordings of tubular pressure from a single nephron in order to compare experimental and simulation results. Normotensive rats exhibit regular oscillations in their tubular pressure. From Fig. 2 (presented in Sec. II together with the method description) it can be seen that the modulation frequency of the fast myogenic oscillations (white circles) is in the same frequency band as the frequency of the TGF-mediated oscillations (black circles).
The tubular pressure data from a hypertensive rat (Fig. 4) are strongly nonstationary. All frequencies identified in the process show essential temporal variations [Figs. 4(b), 4(c) and 4(d)]. Despite this nonstationarity the proposed doublewavelet approach allows us to obtain a qualitative similarity between the dependences of the instantaneous modulation frequencies and the slow rhythm. Note, that "switchings" of the modulation frequencies take place earlier than the corresponding changes of the TGF dynamics. This may be a result of the averaging effect: To estimate the characteristics at a fixed time moment we use a "sliding window" with the width of the wavelet mother function at the chosen frequency, i.e., we consider both some previous and some future dynamics.

Based on these results it is possible to conclude that (i) the proposed approach allows us to estimate the properties of modulation of the fast oscillatory mode extracted from the original miltimode data; and (ii) in accordance with the model, both amplitude and frequency modulation are presented in the experimental data.

The analyses of experimental data presented to this point were made from tubular pressure recordings. Such measurements are rather easy to perform and have relatively low measurement noise. They suffer from the disadvantage that the myogenic oscillations originate in the arterioles and that the fast oscillations in tubular pressure are damped by the compliant nature of the tubule wall, which (together with the associated flow resistances) serves as a mechanical low pass filter. An experimental laser Doppler method for measuring blood flow in a single efferent arterioles on the surface of the kidney $[25,26]$ allows one to observe more pronounced myogenic oscillations compared to those revealed by the tubular pressure recordings, but at the same time contain more measurement noise. At the level of whole kidney blood flow, a Volterra-Wiener kernel analysis has previously revealed nonlinear interactions between the two mechanisms in kidneys 

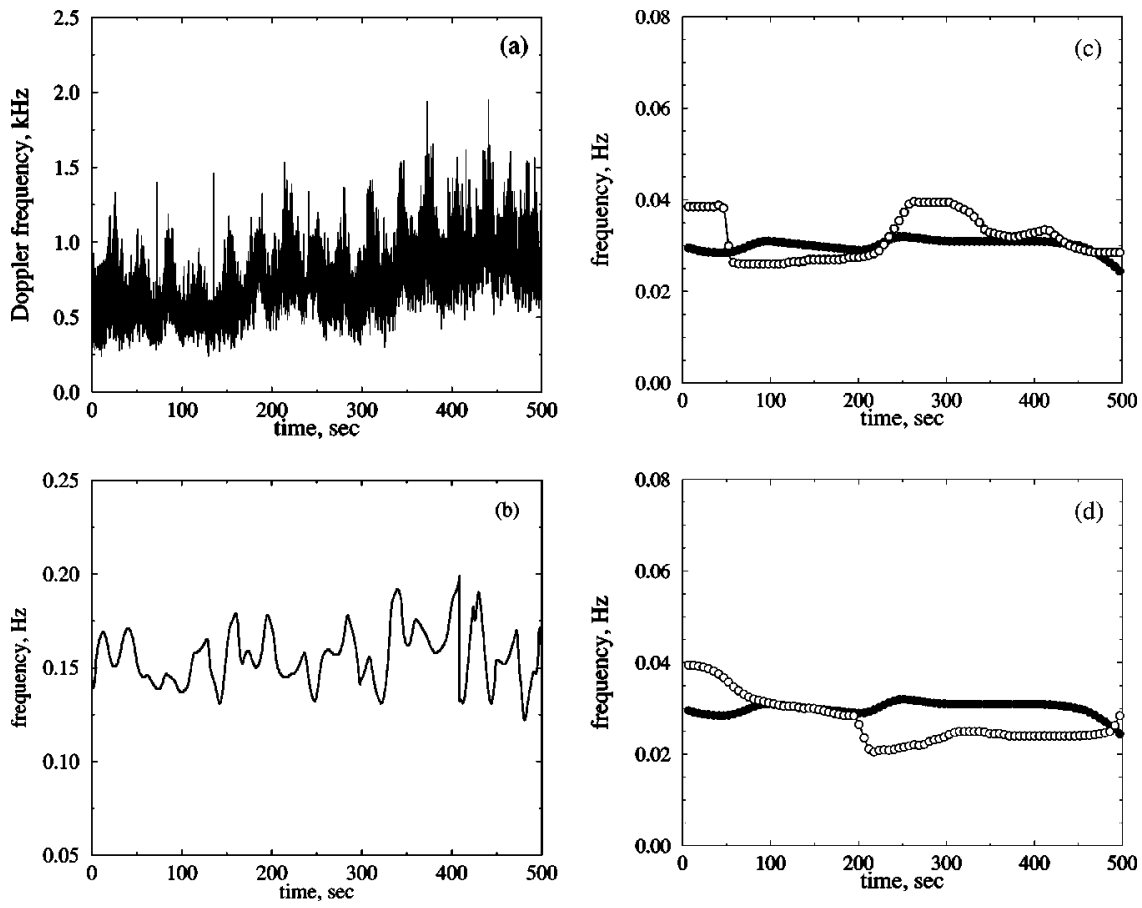

FIG. 5. Analysis of blood flow data from the nephron of a normotensive rat. (a) is the original time series. (b) illustrates the instantaneous frequency $f_{\text {fast }}(t)$. (c) and (d) show $f_{\text {slow }}(t)$ (black circles) and the modulation frequency (white circles) for the frequency and amplitude modulation of the fast mode, respectively. subjected to broad band forcing of the arterial blood pressure [27].

Figures 5 and 6 demonstrate the results obtained for a normotensive and a hypertensive rat, respectively. In both figures black circles denote the temporal dependences of the instantaneous frequencies of the slow oscillatory mode, and white circles are related to the instantaneous modulation frequencies. For a normotensive rat (Fig. 5) the modulation frequency displays some fluctuations around the frequency of the slow oscillatory mode. The variations from the TGF frequency do not exceed $0.01 \mathrm{~Hz}$, which is about the limit of detection of the method.
A similar situation exists for the hypertensive rat (Fig. 6), although the analyzed data are rather nonstationary. Some delay between the instantaneous frequencies [Fig. 6(b)] may arise from the earlier mentioned averaging effect. The modulation frequencies in Figs. 6(c) and 6(d) are close to the slow rhythm, although the rapid changes of the TGF mode (around $t \approx 400 \mathrm{sec}$ ) are not reproduced.

\section{DISCUSSION}

The kidney regulates the incoming blood flow at the level of each nephron through a combination of nonlinear mecha-
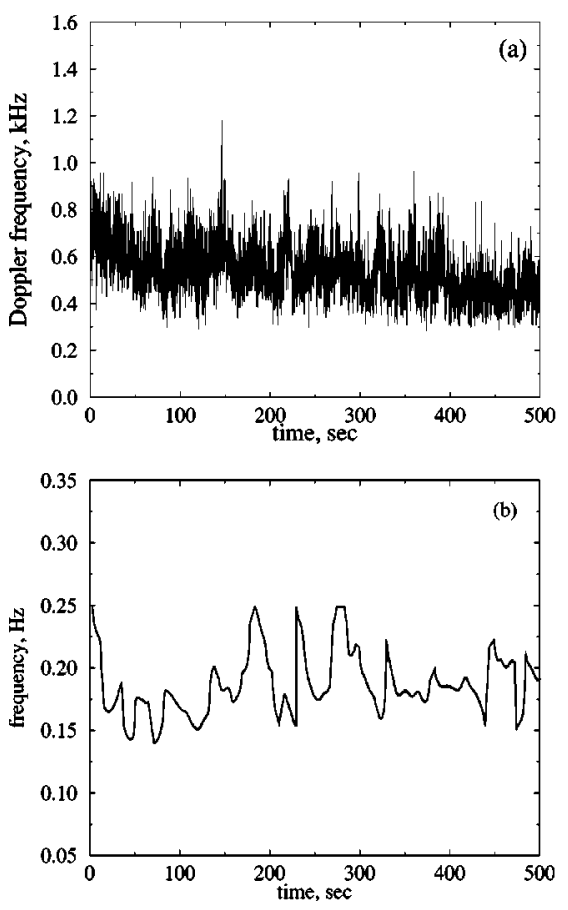
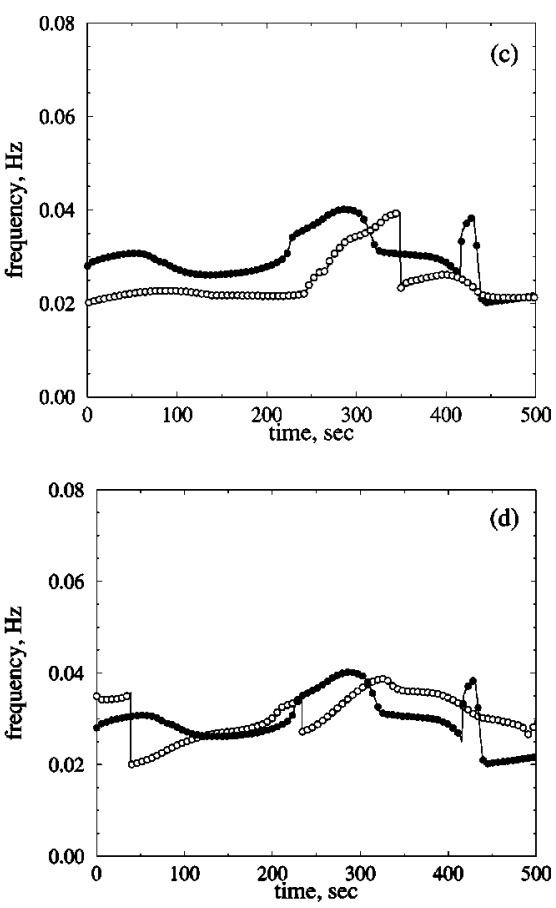

FIG. 6. Analysis of blood flow data from a nephron of a hypertensive rat (a) exhibiting chaotic dynamics. The instantaneous frequency $f_{\text {fast }}(t)$ is shown in (b). (c) frequency modulation and (d) amplitude modulation. The extracted modulation frequencies (white circles) correspond to the frequencies of the slow mode (black circles). 
nisms that generate oscillatory modes of different frequencies. Similar multimode phenomena arise in many other physiological systems and there is a considerable interest in studying the interation between the underlying mechanisms. With the aim of characterizing the modulation properties of the myogenic mode in neprhon autoregulation, we developed a double-wavelet approach that allows us to detect all spectral components involved in the modulation, estimate their contribution, and describe how the modulation properties are changed in response to changes of the system parameters. Using experimental time series for the tubular pressure and arteriolar blood flow, we have shown that these data suffice to estimate the instantaneous modulation frequencies of the fast oscillatory mode.

Comparison of the results of the mathematical model for the single nephron and experimental data allows us to conclude that the frequency and amplitude modulations occur similarly in both cases, i.e., the model reflects the main effects taking place in the real dynamics. We also conclude that the modulation process contains a rhythmic component with the same frequency as the TGF dynamics.

Amplitude modulation of the fast mode by the slow mode has previously been shown [27], but frequency modulation is heretofore unknown in the renal circulation, or in other vascular beds. Frequency modulation represents a form of frequency encoding and provides more robust signaling in a noisy enviroment than can be achieved by amplitude modulation alone. The $1 / f$ fluctuations in arterial pressure have the potential of causing large fluctuations in renal blood flow and therefore in flow rate of tubular fluid. The combined action of TGF and the myogenic mechanism serve to constrain the resultant fluctuations in renal blood flow, but it is important that the two mechanisms act cooperatively to limit fluctuations of the mass flow of $\mathrm{NaCl}$ to the distal nephron whose epithelial transport functions operate under hormonal control.

Extraction of the instantaneous frequencies from the surface $E_{x}(f, t)$ can sometimes lead to problems in determination of the points of the instantaneous frequency. In particular, this may be a case when a fast mode is comparable in power to the harmonics of the slow mode and they are close in the frequency domain. In such situations, we may lose some part of the modulation period while extracting $f_{\text {fast }}(t)$ for the remaining part. Analysis of $f_{\text {fast }}(t)$ will therefore allow estimation of the modulation frequency (losing some points can influence only the harmonics which is not important for the aims of our study). However, in the last case it will be difficult to define the modulation depth. Of course, such problems do not always arise, we would like only to note that they may occur. Actually, to make correct conclusions about the modulation depth in the nephron dynamics we need at least two conditions to be satisfied: (i) the harmonics of the slow mode (third harmonic and higher) should be small in power in comparison with the fast mode (this is probably the case for real nephron dynamics where these harmonics are usually less expressed than in the model); and (ii) the data should be reasonably stationary. This means that it should be possible from the experiments to obtain time series such that the modulation depth can be estimated (especially in the case of regular dynamics).

\section{ACKNOWLEDGMENTS}

This work was partly supported by INTAS (Grant No. 01-2061) and by the Danish National Science Foundation. O.S. acknowledges support from the Lundbeck Foundation. A.P. acknowledges support from $\mathrm{CRDF}$ within the framework of the BRHE Program (Award No. SR-006-X1 and 2003 Post-Doctoral Fellowship Award No. Y1-P-06-06) and from RFBR (Grant No. 04-02-16769).
[1] U. Hoffman, A. Yanar, U.K. Franzeck, J.M. Edvards, and A. Bollinger, Microvasc. Res. 40, 293 (1990).

[2] N.-H. Holstein-Rathlou, J. He, A.J. Wagner, and D.J. Marsh, Am. J. Physiol. 269, F1230 (1995).

[3] A. Goldbeter, Biochemical Oscillations and Cellular Rhythms (Cambridge University Press, Cambridge, 1996).

[4] X.-J. Wang and J. Rinzel, in The Handbook of Brain Theory and Neural Networks, edited by M. A. (MIT Press, Cambridge, 1995).

[5] H.A. Braun, H. Bade, and H. Hensel, Pfluegers Arch. 386, 1 (1980).

[6] J. Sturis, E. Mosekilde, and E. Van Cauter, Methods Neurosci. 20, 393 (1994).

[7] C. Schäfer, M.G. Rosenblum, J. Kurths, and H.-H. Abel, Nature (London) 392, 239 (1998).

[8] N. Kopell, G.B. Ermentrout, M.A. Whittington, and R.D. Traub, Proc. Natl. Acad. Sci. U.S.A. 97, 1867 (2000).

[9] E. Mosekilde, Topics in Nonlinear Dynamics: Applications to Physics, Biology and Economic Systems (World Scientific, Singapore, 1996).

[10] D.E. Postnov, O.V. Sosnovtseva, E. Mosekilde, and N.-H.
Holstein-Rathlou, Int. J. Mod. Phys. B 15, 3079 (2001).

[11] N.-H. Holstein-Rathlou, K.-P. Yip, O.V. Sosnovtseva, and E. Mosekilde, Chaos 11, 417 (2001).

[12] O.V. Sosnovtseva, A.N. Pavlov, E. Mosekilde, and N.-H. Holstein-Rathlou, Phys. Rev. E 66, 061909 (2002).

[13] M.M. Wolf, G.A. Varigos, D. Hunt, and J.G. Sloman, Med. J. Aust. 2, 52 (1978).

[14] A. Grossmann and J. Morlet, SIAM J. Math. Anal. 15, 723 (1984); I. Daubechies, Ten Lectures on Wavelets (SIAM, Philadelphia, 1992).

[15] G. Kaiser, A Friendly Guide to Wavelets (Boston, Birkhäuser, 1994).

[16] C.-K. Peng, S. Havlin, H.E. Stanley, and A.L. Goldberger, Chaos 5, 82 (1995).

[17] J.F. Muzy, E. Bacry, and A. Arneodo, Int. J. Bifurcation Chaos Appl. Sci. Eng. 4, 245 (1994).

[18] P. Tass, M.G. Rosenblum, J. Weule, J. Kurths, A. Pikovsky, J. Volkmann, A. Schnitzler, and H.-J. Freund, Phys. Rev. Lett. 81, 3291 (1998).

[19] M. Holschneider, R. Kronland-Martinet, J. Morlet, and A. Grossmann, in Wavelets: Time-Frequency Methods and Phase 
Space, edited by J.M. Combes, A. Grossmann, and $\mathrm{Ph}$. Tchamitchian (Springer, Berlin, 1989), p. 286; R. Quian Quiroga, A. Kraskov, T. Kreuz, and P. Grassberger, Phys. Rev. E 65, 041903 (2002).

[20] N.-H. Holstein-Rathlou and P.P. Leyssac, Acta Physiol. Scand. 126, 333 (1986).

[21] P.P. Leyssac and N.-H. Holstein-Rathlou, Pfluegers Arch. 407, 285 (1986).

[22] N.-H. Holstein-Rathlou and D.J. Marsh, Physiol. Rev. 74, 637 (1994).

[23] M.D. Andersen, N. Carlson, E. Mosekilde, and N.-H. Holstein-
Rathlou, in Membrane Transport and Renal Physiology, edited by H. Layton and A. Weinstein (Springer-Verlag, New York, 2001).

[24] M. Barfred, E. Mosekilde, and N.-H. Holstein-Rathlou, Chaos 6, 280 (1996).

[25] G.T. Smedley, K.P. Yip, A.J. Wagner, S. Dubovitsky, and D.J. Marsh, IEEE Trans. Biomed. Eng. 40, 290 (1993).

[26] K.P. Yip, N.-H. Holstein-Rathlou, and D.J. Marsh, Am. J. Physiol. 262, F980 (1992).

[27] Ki H. Chon, Y.-M. Chen, V.Z. Marmarelis, D.J. Marsh, and N.-H. Holstein-Rathlou, Am. J. Physiol. 267, F160 (1994). 